Supplement of

\title{
Aerosol characteristics and particle production in the upper troposphere over the Amazon Basin
}

Meinrat O. Andreae et al.

Correspondence to: Meinrat O. Andreae (m.andreae@mpic.de)

The copyright of individual parts of the supplement might differ from the CC BY 4.0 License. 
Figure S1: Example of the center of a back-tracked parcel starting at the location of the HALO aircraft during flight AC18. This parcel started at 2014-09-28 20:34:53 UTC (same time as the HALO measurement) and was traced back in time in one-hour steps up to 120 hours, as shown in the time color scale on the right. The solid gray line is the full flight track of AC18. The top panel shows longitude vs. latitude and the bottom panel shows the longitude vs. altitude positions. We can see that this parcel stays in the UT within the 120 hours considered.

Flight AC18 - LegE1

Parcel start @ 2014-09-28 20:34:53

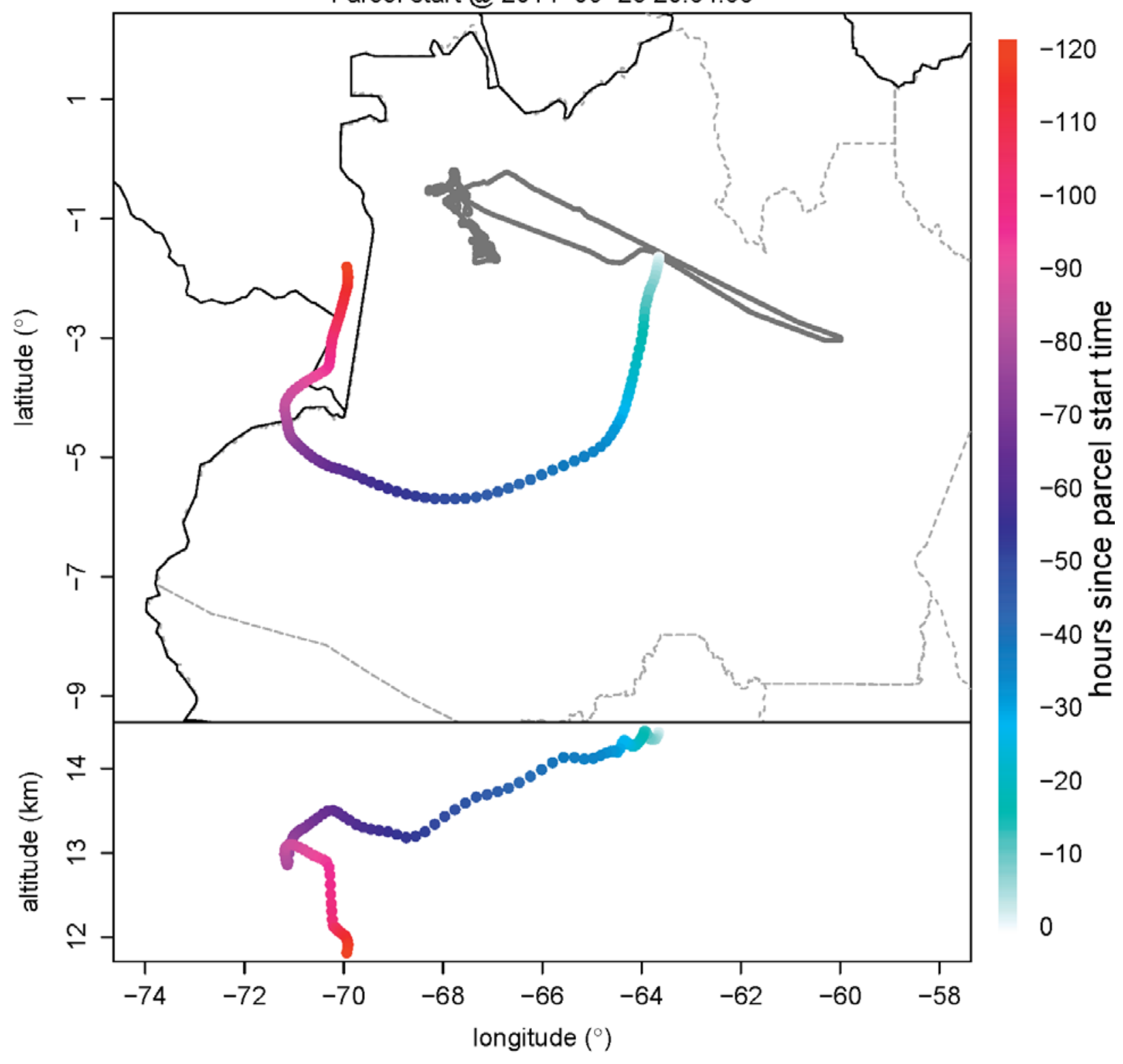


Figure S2: Examples of snapshots of the back-tracked parcel shown in Figure S1 matched in time to the closest GOES-13 infrared brightness temperature $\left(T_{b}\right)$. Color dots are the same ones as in Figure S1, representing the position and backward time (in hours). Here the snapshots are zoomed in a $6^{\circ} \times 6^{\circ}$ box centered at the parcel location at the time shown on the top the snapshots and the number in parentheses is the number of hours backward from the parcel start (and flight measurement). Dashed boxes show the $1^{\circ} \times 1^{\circ}$ box centered in the back-tracked parcel within which we looked up the minimum Tb. This value is shown at the left bottom corner of each snapshot as well as the parcel altitude (in $\mathrm{m}$ ) and GOES-13 infrared (IR) time.
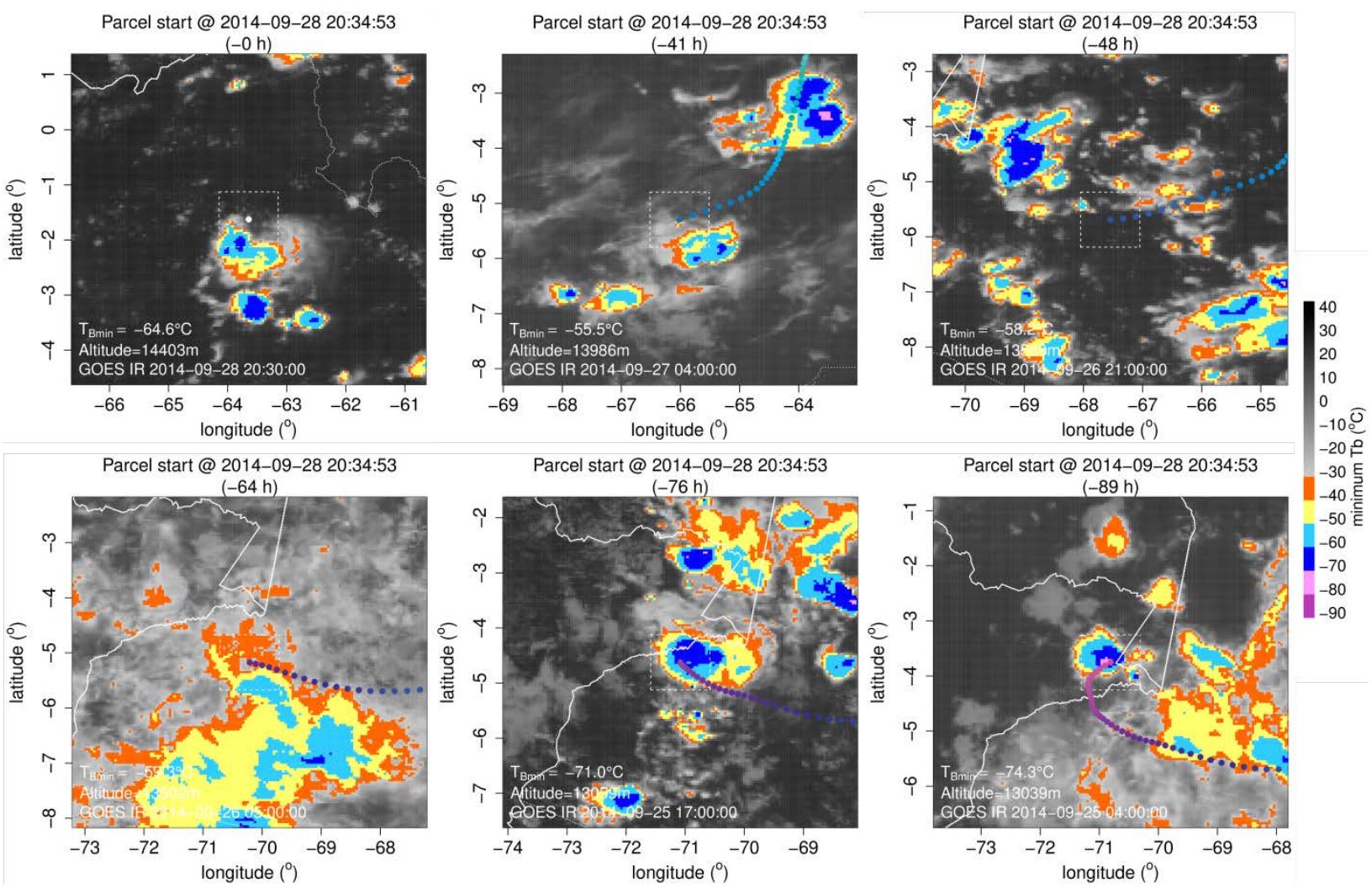
Figure S3: Summary of the back-tracked parcel positions (as in Fig. S1) with the minimum $T_{b}$ tracked as shown in the snapshot examples of Figure S2. Here the back-track parcel position is colored with the value of minimum $T_{b}$ found in the $1^{\circ} \times 1^{\circ}$ box.

Flight AC18 - LegE1

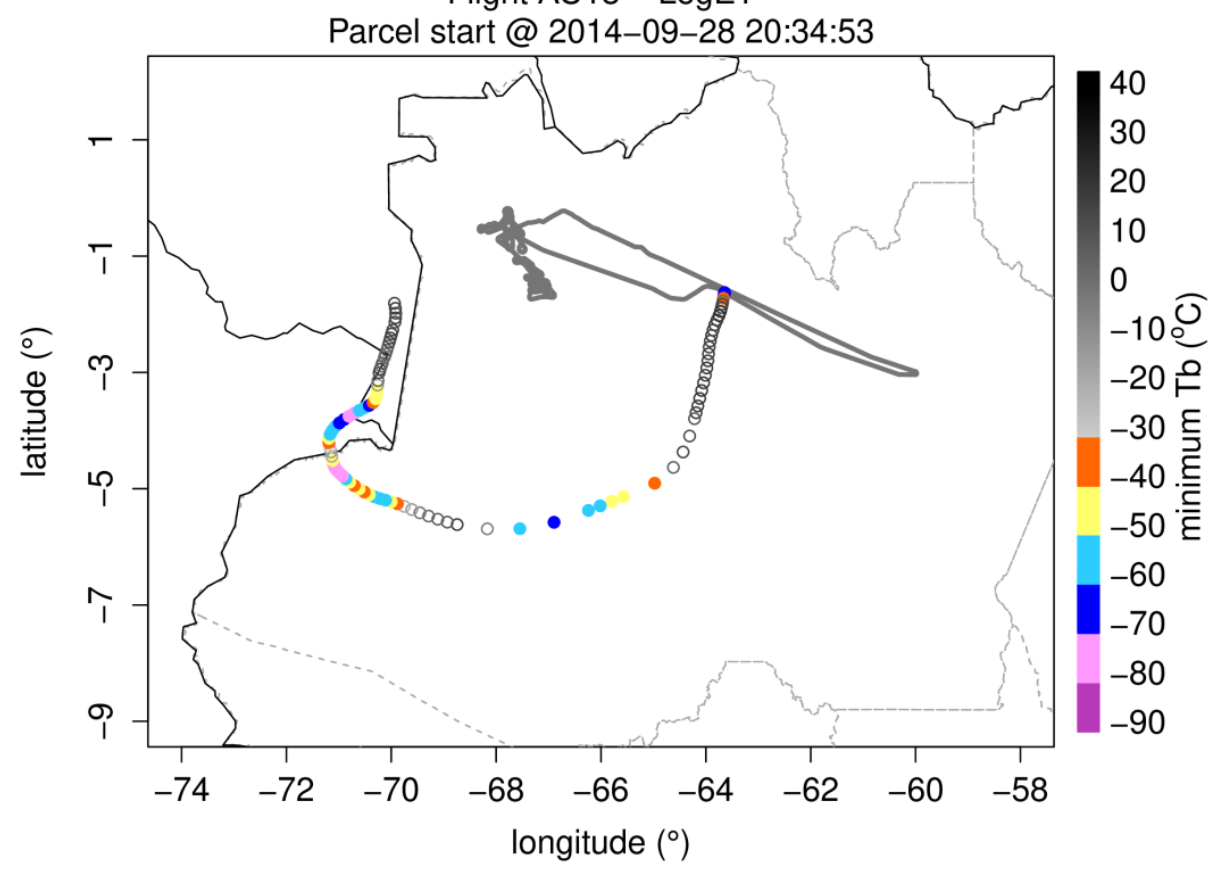


Figure S4. Mean vertical profiles of temperature, relative humidity and potential temperature $(\theta)$ from radiosoundings at Manacapuru.
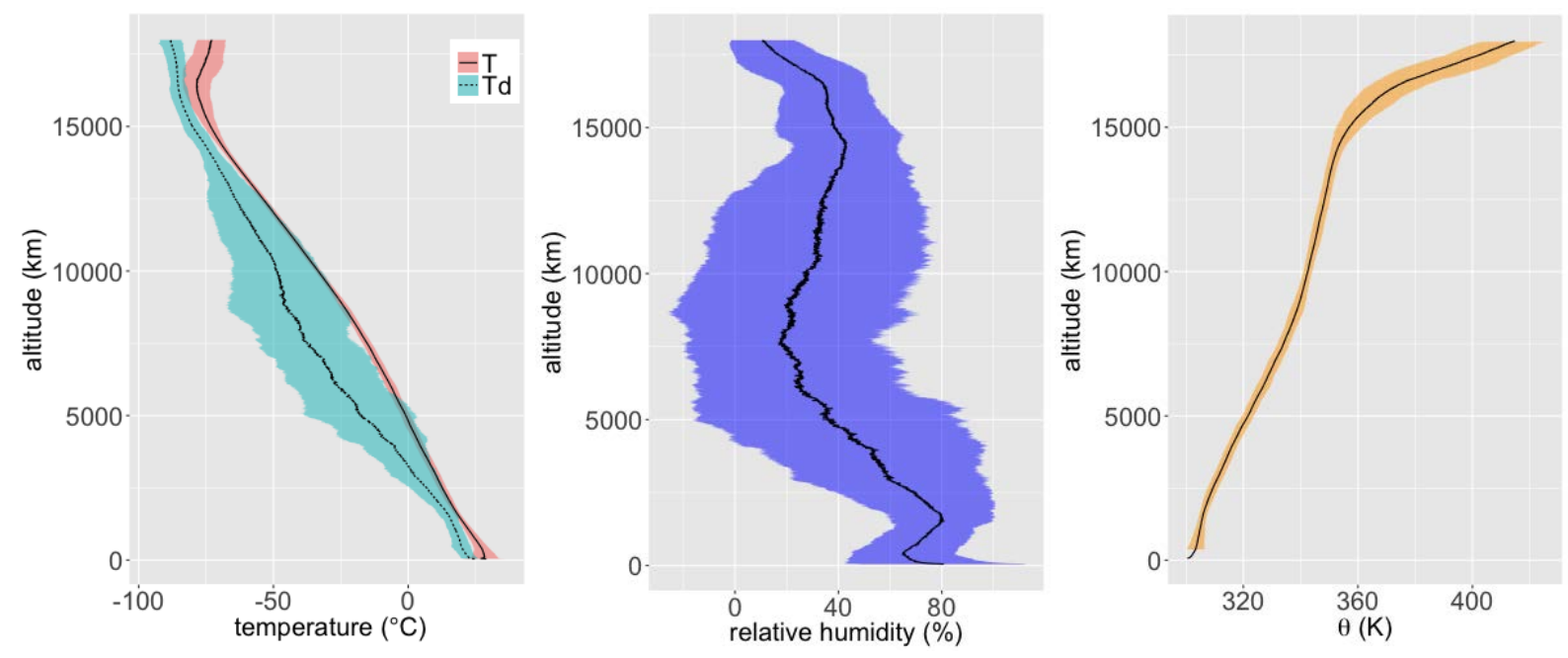
Figure S5: Trajectory statistics based on 120-hour backtrajectory calculations for September 2014, initialized at Manaus at an elevation of $10 \mathrm{~km}$.

NOAA HYSPLIT MODEL - TRAJECTORY FREQUENCIES

\# endpts per grid sq./\# trajectories (\%) $0 \mathrm{~m}$ and $99999 \mathrm{~m}$ Integrated from 210030 Sep to 090027 Aug 14 (UTC) [backward] Freq Calculation started at $000000 \quad 00$ (UTC)

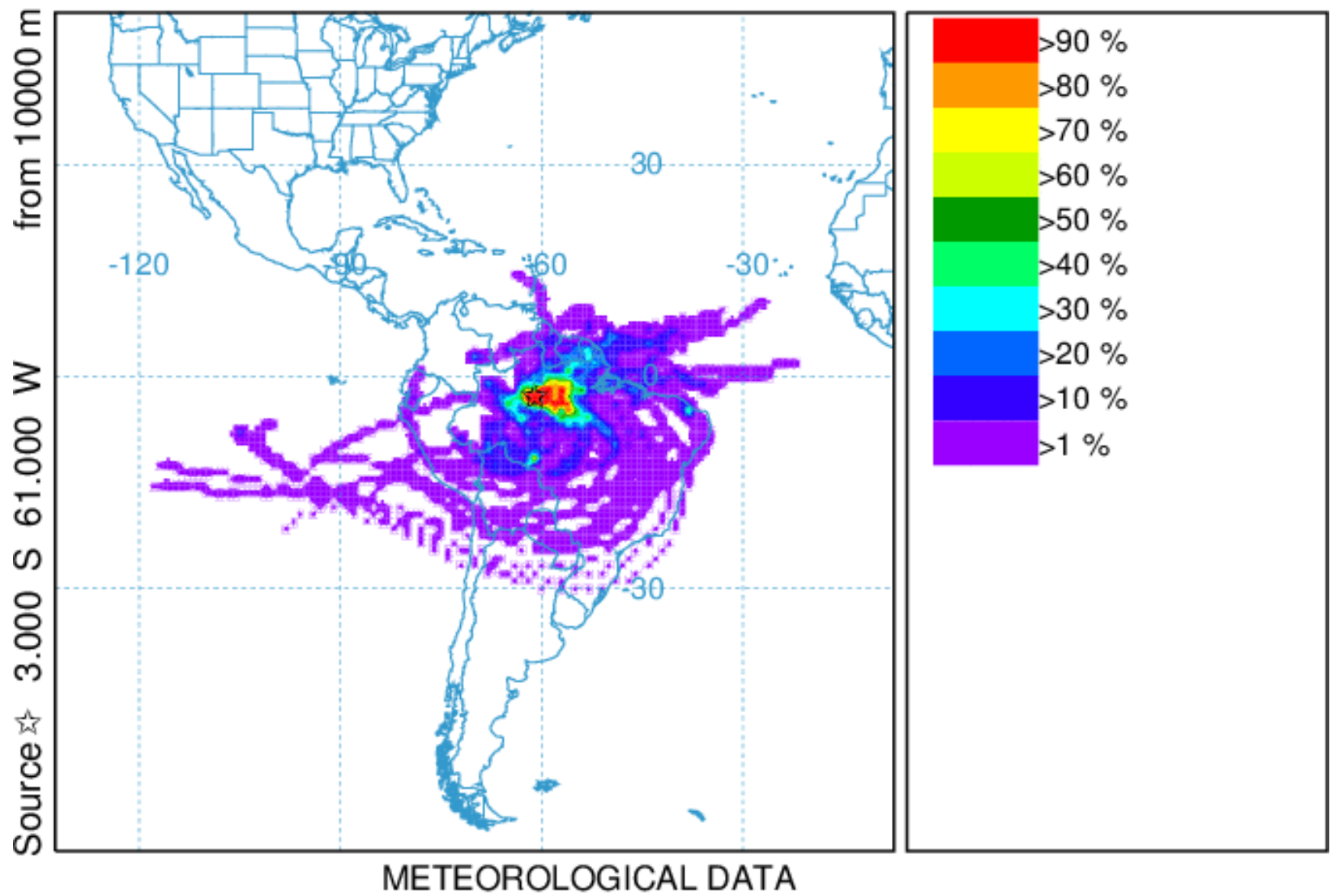

Job ID: $113173 \quad$ Job Start: Wed Jan 11 22:11:28 UTC 2017

Source 1 lat:: -3.000000 lon.: -61.000000 height: $10000 \mathrm{~m}$ AMSL

Initial trajectory started: $2100 Z 30$ Sep 14

Direction of trajectories: Backward Trajectory Duration: $120 \mathrm{hrs}$

Frequency grid resolution: $1.0 \times 1.0$ degrees

Endpoint output frequency: 60 per hour

Number of trajectories used for this calculation: 60

Meteorology: 0000Z 29 Sep 2014 - GDAS1 
Figure S6: Trajectory statistics based on 120-hour backtrajectory calculations for September 2014, initialized at Manaus at an elevation of $14 \mathrm{~km}$.

\section{NOAA HYSPLIT MODEL - TRAJECTORY FREQUENCIES}

\# endpts per grid sq./\# trajectories (\%) $0 \mathrm{~m}$ and $99999 \mathrm{~m}$ Integrated from 210030 Sep to 090027 Aug 14 (UTC) [backward] Freq Calculation started at $000000 \quad 00$ (UTC)

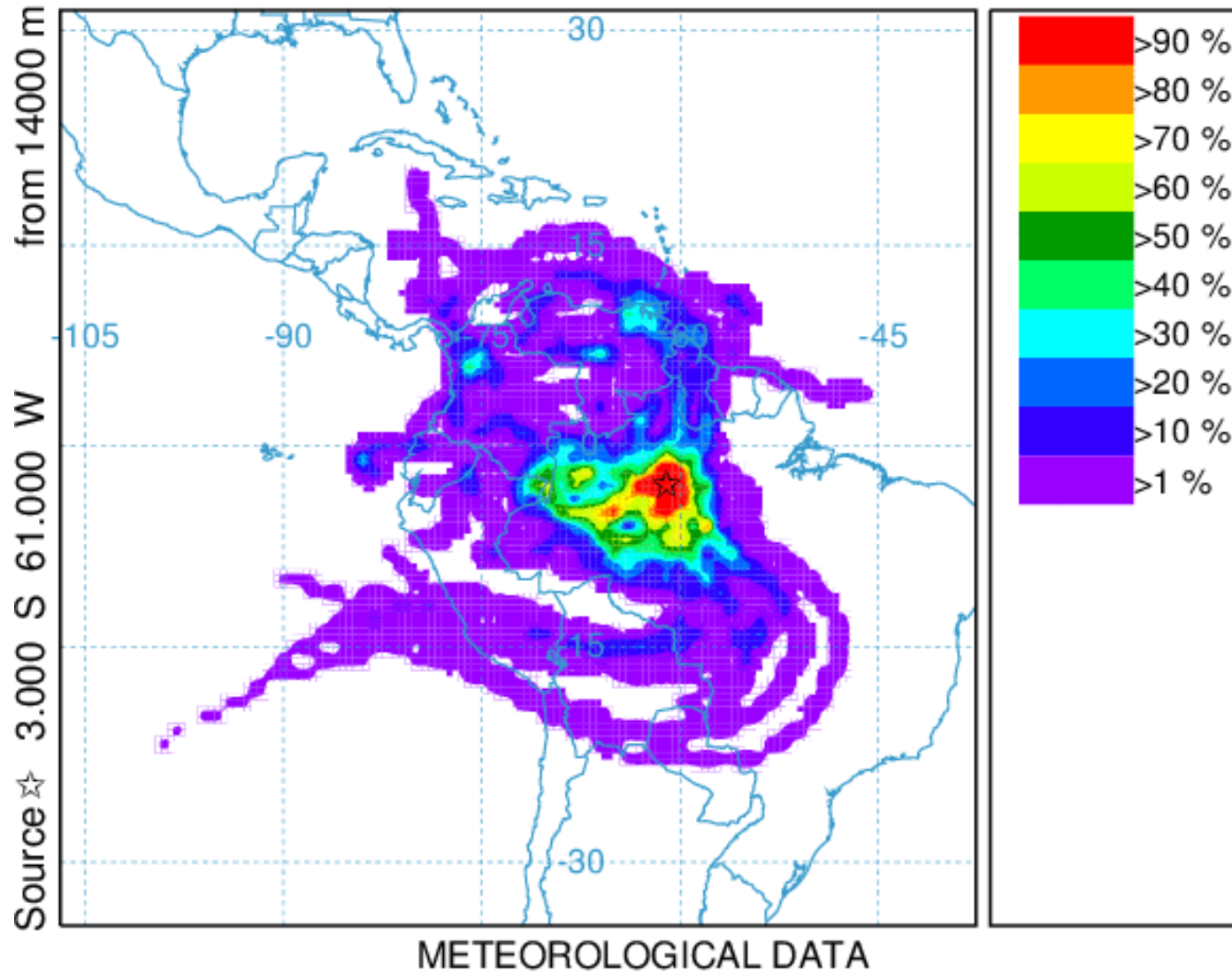

Job ID: 113224

Job Start: Wed Jan 11 22:18:03 UTC 2017

Source 1 lat.: -3.000000 lon.: -61.000000 height: $14000 \mathrm{~m}$ AMSL

Initial trajectory started: $2100 Z 30$ Sep 14

Direction of trajectories: Backward Trajectory Duration: $120 \mathrm{hrs}$

Frequency grid resolution: $1.0 \times 1.0$ degrees

Endpoint output frequency: 60 per hour

Number of trajectories used for this calculation: 60

Meteorology: 0000Z 29 Sep 2014 - GDAS1 
Figure S7: Time series plot of $\mathrm{CN}$ and $\mathrm{CCN}(0.52 \%)$ concentrations and flight altitude from flight AC09.

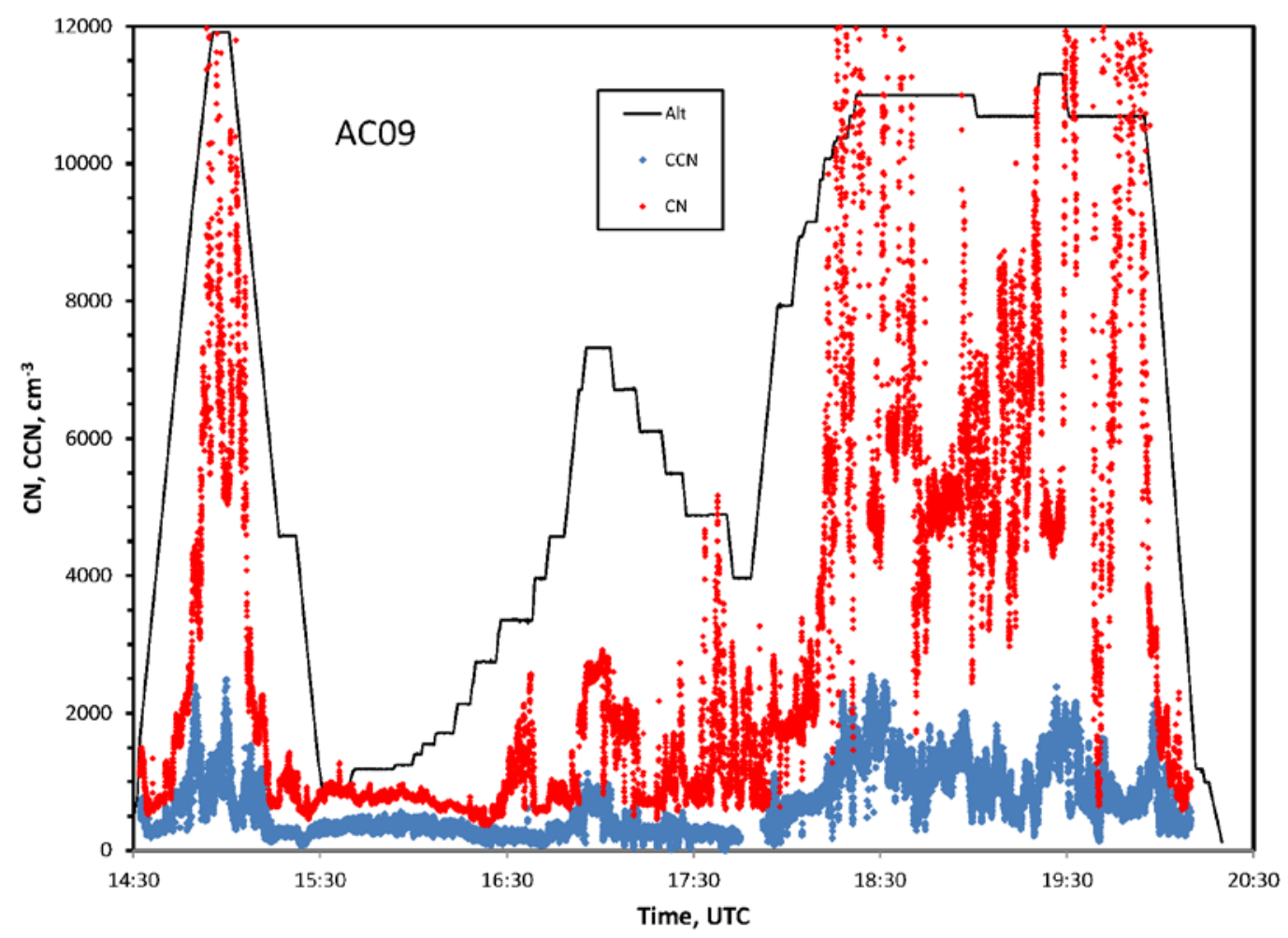


Figure S8: Scatter plot of $\mathrm{CN}$ vs $\mathrm{O}_{3}$ concentrations from the UT in flight AC09.

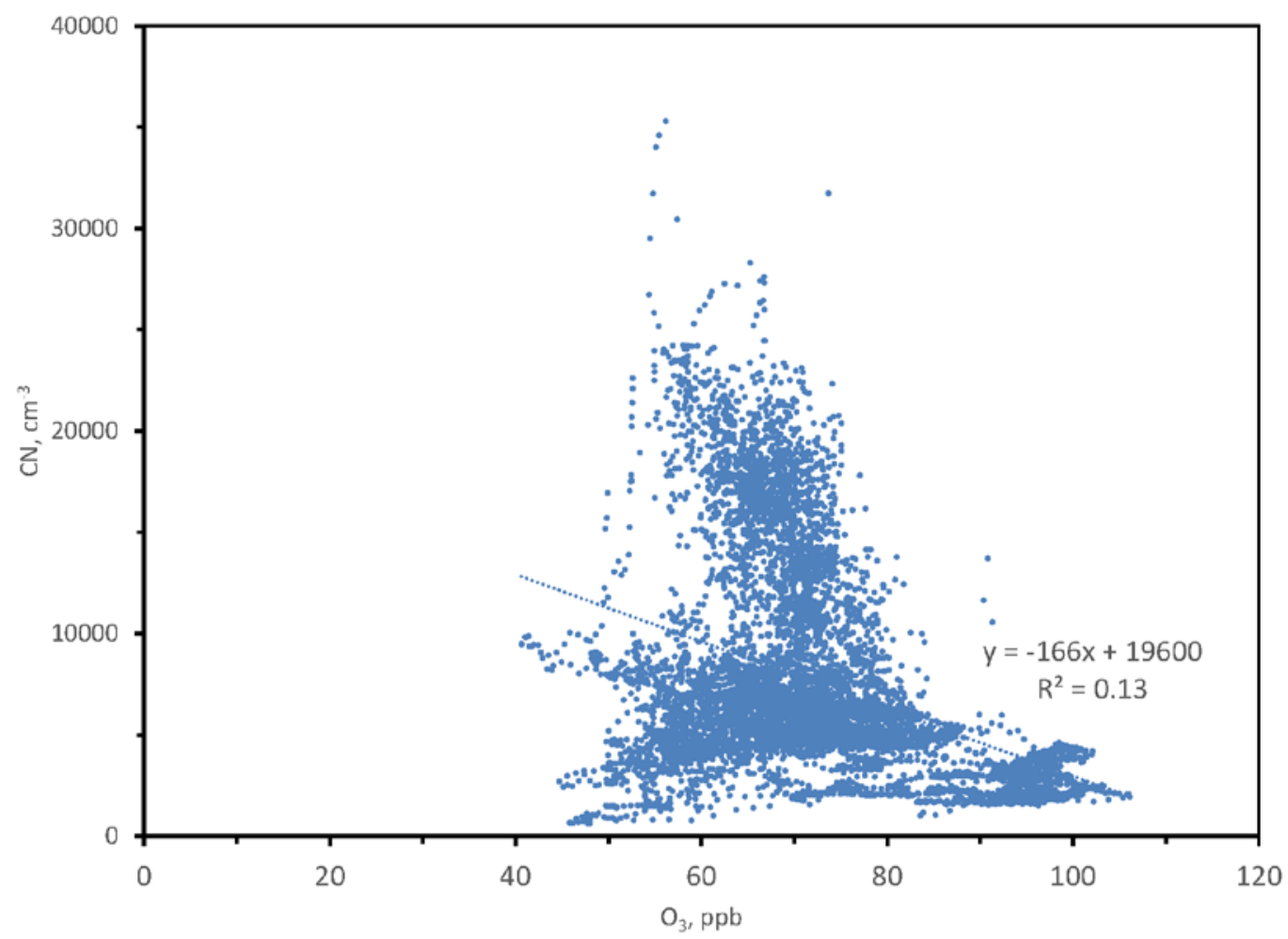


Table S1: Properties of the flight legs on which low aerosol concentrations were measured in the UT during ACRIDICON-CHUVA.

\begin{tabular}{|c|c|c|c|c|c|c|c|c|c|c|c|c|c|c|}
\hline Flight & Leg & $\begin{array}{l}\text { Start } \\
\text { UTC }\end{array}$ & $\begin{array}{l}\text { End } \\
\text { UTC }\end{array}$ & $\begin{array}{c}\text { Altitude } \\
\text { range } \\
\text { m }\end{array}$ & $\begin{array}{l}\mathrm{N}_{\mathrm{CN}} \\
\max . \\
\mathrm{cm}^{-3}\end{array}$ & $\begin{array}{l}\mathrm{N}_{\mathrm{CN}} \\
\text { mean } \\
\mathrm{cm}^{-3}\end{array}$ & $\begin{array}{c}\text { NCCN0.5 } \\
\text { mean } \\
\mathrm{cm}^{-3}\end{array}$ & $\begin{array}{l}\mathrm{Nacc} \\
\text { mean } \\
\mathrm{cm}^{-3}\end{array}$ & $\begin{array}{l}\text { Ultrafine } \\
\text { fraction }\end{array}$ & $\begin{array}{l}\text { Trajectory } \\
\text { type }\end{array}$ & $\begin{array}{c}\operatorname{Min} \mathrm{T}_{\mathrm{b}} \\
{[\min , \mathrm{max}]^{\mathrm{a}}} \\
{ }^{\circ} \mathrm{C}\end{array}$ & $\begin{array}{c}\text { Time since last } \\
\text { DC }[\text { min,max }]^{\mathrm{b}} \\
\text { hours }\end{array}$ & $\begin{array}{c}\text { Time in DC } \\
{[\text { min,max }]^{\mathrm{c}}} \\
\text { hours }\end{array}$ & $\begin{array}{l}\text { Sampling } \\
\text { environment }\end{array}$ \\
\hline AC16 & $\mathrm{L}$ & $16: 23$ & $16: 30$ & $12200-12600$ & 3840 & 2460 & 169 & 142 & 0.94 & B & {$[-62,-40]$} & {$[17,18]$} & {$[5,11]$} & clear air \\
\hline AC18 & $\mathrm{L}$ & $15: 17$ & $15: 19$ & $9000-10500$ & 1980 & 1620 & 487 & 236 & 0.85 & $\mathrm{C}$ & {$[-2,4.6]$} & - & - & clear air \\
\hline AC20 & L1 & $19: 21$ & $19: 23$ & $13600-13900$ & 3040 & 1460 & 291 & - & - & A & {$[-75,-70]$} & {$[0,0]$} & {$[14,16]$} & clear air near outflow \\
\hline AC20 & L2 & 19:33 & 19:35 & 14400 & 2190 & 1350 & 300 & - & - & A & {$[-62,-61]$} & {$[0,0]$} & {$[10,12]$} & clear air near outflow \\
\hline AC20 & L3 & 19:52 & 19:57 & 14100 & 1950 & 1200 & 256 & - & - & A & {$[-67,-68]$} & {$[0,0]$} & {$[10,13]$} & clear air near outflow \\
\hline
\end{tabular}

a) Minimum and maximum temperature at top of most recent deep convection in grid boxes through which the center trajectories for the flight leg had passed.

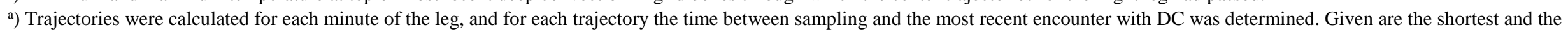
longest of these time intervals.

a) Minimum and maximum length of time that the trajectories from each leg had spent in grid boxes with DC. 